TELKOMNIKA, Vol.16, No.1, February 2018, pp. 134 141

ISSN: 1693-6930, accredited A by DIKTI, Decree No: 58/DIKTI/Kep/2013

DOI: 10.12928/TELKOMNIKA.v16i1.9025

\title{
A Solution to Partial Observability in Extended Kalman Filter Mobile Robot Navigation
}

\author{
Hamzah Ahmad*, Nur Aqilah Othman, Mohd Syakirin Ramli \\ Faculty of Electrical \& Electronics Engineering,Universiti Malaysia Pahang, Pekan Campus, 26600 Pekan, \\ Pahang Darul Makmur, 09-4246024, Malaysia \\ *Corresponding author, e-mail: hamzah@ump.edu.my
}

\begin{abstract}
Partial observability in EKF based mobile robot navigation is investigated in this paper to find a solution that can preventerroneous estimation. By only considering certain landmarks in an environment, the computational cost in mobile robotcan be reduced but with an increase of uncertainties to the system. This is known as suboptimal condition of the system. Fuzzy Logic technique is proposed to ensure that the estimation achieved desired performance even though some of the landmarks were excluded for references. The Fuzzy Logic is applied to the measurement innovation of Kalman Filter to correct the positions of both mobile robot and any observed landmarks during observations. The simulation results shown that the proposed method is capable to secure reliable estimation results even a number of landmarks being excluded from Kalman Filter update process in both Gaussian and non-Gaussian noise conditions.
\end{abstract}

Keywords: partial observability, mobile robot, navigation, fuzzy logic, estimation

Copyright (C) 2018 Universitas Ahmad Dahlan. All rights reserved.

\section{Introduction}

Mobile robot navigation is one of the fascinating research which supports a truly autonomous mobile robot behavior. It is one of the aspects that really means in nowadays applications especially when a robot needs to perform a task to replace human operation. Robot has been known to functionally works in explorations, underwater navigation, mining and military by performing different kind of tasks. Depends on the applications, the mobile robot may require several conditions to be satisfied about its design and specifications. In navigation, one of its challenges is known as Simultaneous Localization and Mapping problem where mobile robot has to identify its location with reference to any of landmarks being observed and at the same time building a map based on its observations. This problem addressed several issues to be solved such as operating in dynamic environment, computational cost, uncertainties and data association [1]-[2].

Three main approaches are available in SLAM which are the mathematical modeling, the behavioral model and the probabilistic techniques. Among these three popular methods, probabilistics has captured most of the researcher interest where a lot of techniques have been proposed based on probabilistics [1]. EKF as one of the celebrated probabilistics approach in SLAM made a significant achievement as it is the most successful method to be applied online. In EKF based navigation, there a some issues to be solved and one of it is the computational cost. The computational cost increased if more landmarks are observed and processed during mobile robot movements. However, this problem do not influence the estimation performance but instead will require higher cost to build the robot.

This paper analyzes the aspect of partial observability as one of the problem in EKF based SLAM [3]-[4]. A sensor such as infrared sensor, sonar sensor or laser range finder attached to the mobile robot can obtained information about the environment regarding its relative measurements i.e relative angle and distance. The information generally contains various data. Unfortunately, these data can be erroneous due to unexpected conditions. This may due to the sensor fault or system limitations that a mobile robot is equipped with. Owing to these circumstances, mobile robot can accidentally lost its way and cannot perform localization and mapping accurately. Interestingly, this problem sometimes is very difficult to be recognized as mobile robot can intermittently experience such problem without prior notice. One way to 
demonstrate this condition is by considering a mobile robot which is able to recognize several landmarks at a time compared to other different observation time. Even though this problem is critical, still under investigation and further improvement, the situation has motivates a case to determine the system performance when only several identified landmarks are referred during mobile robot observations. By doing this, it is expected that the system can reduce its computational cost and at the same time guarantees good estimation results [5], [6].

In SLAM, the partial observability problem is categorized and analyzed into three main aspects; unstable partially observable, partially observable and observable SLAM problem[7]. It is claimed that the partially observability has makes the mobile robot depends heavily on its initial conditions [8], [9]. Due to partial observability, the performance of observation can become worse as the updated state covariance of the system cannot guaranteed to be converge even if the mobile robot has done multiple observations on its surroundings. EKFSLAM has been one of the influential technique and been analyzed thoroughly in different perspectives [10]-[13]. The technique is also been widely applied in real-life applications for navigation purposes.

This paper is organized as follow. Section 2 describes the proposed technique of EKF based SLAM and Fuzzy Logic technique. Section 3 demonstrates the simulation results and analysis of the proposed method amd finally section 4 concludes the paper.

\section{Extended Kalman Filter and Fuzzy Logic Approaches in SLAM}

\subsection{Extended Kalman Filter in SLAM}

SLAM normally consists of two general processes which are the kinematics or process step and the measurement step. The process stage defines the mathematical analysis of the model focusing on the kinematics of the mobile robot with consideration of the landmarks location being recognized. SLAM uses the mobile robot velocity and angular velocity to determine its location. The measurement step on the other hand calculates the relative angle and distance between mobile robot and any observed landmarks. Both of process and measurement stages utilizes the mobile robot sensors to determine the positions and map building. The process step is as follows.

$$
\mathrm{X}_{\mathrm{k}}=\mathrm{AX}_{\mathrm{k}-1}+\mathrm{BU}_{\mathrm{k}-1}+\omega_{\mathrm{k}-1}
$$

where $X_{k}$ is an augmented state consisting of the mobile robot heading angle and $x, y$ positions with landmarks $x, y$ locations. $A$ and $B$ are the transition matrix and control matrix respectively. $U$ is the control input of mobile robot velocity and angular velocity, while $\omega$ is the process noise of the mobile robot. The measurement process takes the following mathematical equation.

$$
\mathrm{Z}_{\mathrm{k}}=\mathrm{HX}_{\mathrm{k}-1}+\mathrm{v}_{\mathrm{k}-1}
$$

$\mathrm{Z}_{\mathrm{k}}$ is the measurement matrix containing information about relative distance and relative angle between mobile robot to each landmark. $\mathrm{H}$ is the measurement matrix and $\mathrm{v}$ is the measurement noise occurred during mobile robot observations. EKF works based on the above two SLAM model to estimate the mobile robot and landmarks positions. There are two main stages to be computed which are predict and update stages. Table 1 shows the EKF steps to perform the estimation. Tables and Figures are presented center, as shown below and cited in the manuscript.

\begin{tabular}{|c|c|c|}
\hline & & Kalman Filter \\
\hline Prediction & State model & $\widehat{X}_{k}=A \widehat{X}_{k-1}+B U_{k-1}$ \\
\hline \multirow{3}{*}{ Update } & State model & $\hat{X}^{+}=A \hat{X}^{-}+K\left(Z_{k}-H \hat{X}^{-}\right)$ \\
\hline & Stata nnarianno & 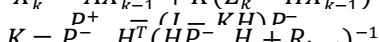 \\
\hline & & $K=P_{k+1} H^{1}\left(H P_{k+1} H\right.$ \\
\hline
\end{tabular}

Table 1. Kalman Filter Algorithm 
As shown in Table 1 above, $\widehat{\mathrm{X}}$ is the estimated augmented state. $\mathrm{P}$ is the state covariance while the sign of "+", "-" defines the priori and posteriori estimation at time k. Kalman Gain plays important role in Kalman Filter where it leads to better estimation. Remarks that, A, $\mathrm{B}, \mathrm{H}$ are the linearized matrices as this study considers EKF as it filter for estimation. The process noise and measurement noise covariances are presented by $Q$ and $R$ and these two noises are uncorrelated throughout the analysis.

From Table 1 , the updated state model consists of element $\mathrm{Z}_{\mathrm{k}}-\mathrm{H} \widehat{\mathrm{X}}_{\mathrm{k}-1}^{-}$which determines the measurement innovation of the system. If the value of this measurement innovation is too big, then Kalman Gain attempts to reduce it so that the updated state model remains holding a very small error during mobile robot observations. From above table 1, this is also means that the measurement innovation must be kept very small at all time. In normal EKF, this cannot be guarantees at all time as sometimes due to environment conditions, there is a possibility of sensor fault which in turns exhibits higher error to the readings. As one of the family of Bayes Filter, EKF only depends on its latest update. Hence, the estimation may becomes erroneous if no further action being made to the system. Having said that, Fuzzy Logic is being proposed to control the amount of information gathered by the sensor. If the measurement innovation accidentally produced higher amount of noises, then the Fuzzy Logic reduced it for better estimation results.

\subsection{Fuzzy Logic Technique}

As mentioned on previous subsection, Fuzzy Logic is proposed to reduced the noises or error developed by the measurement innovation computations. Figure 1 presents the design of Fuzzy Logic to the proposed system. To design the Fuzzy Logic, there are three main phases to be considered i.e Fuzzification, Rule Evaluation and Defuzzification[14]. Fuzzification describes the conditions that needs to be assessed which are in this case the measured relative angle and measured relative distances between mobile robot and landmarks. It is essential to have prior knowledge on the system performance before beginning to design Fuzzy Logic. The input and output of Fuzzy Logic is same as the main objectives of Fuzzy Logic is to reduce the measurement innovation. By knowing these situation, then the fuzzy sets and membership function are selected. Mamdani technique is chosen with three fuzzy sets each for both input and output.

The rule evaluation determines the best configuration of the designed rule to the whole system. It computes the fuzziness of the system based on the rules. Defuzzification is then evaluates using Center of Gravity to obtain the reduced measurement innovation to be calculated with Kalman Gain in achieving small error to the updated system. The fuzzy sets and triangular memberships are included in Figure 2 for references. Triangular membership was selected as it compute faster and regularly used for design compared to other available membership types. The fuzzy sets are defined based on preliminary results of simulations.

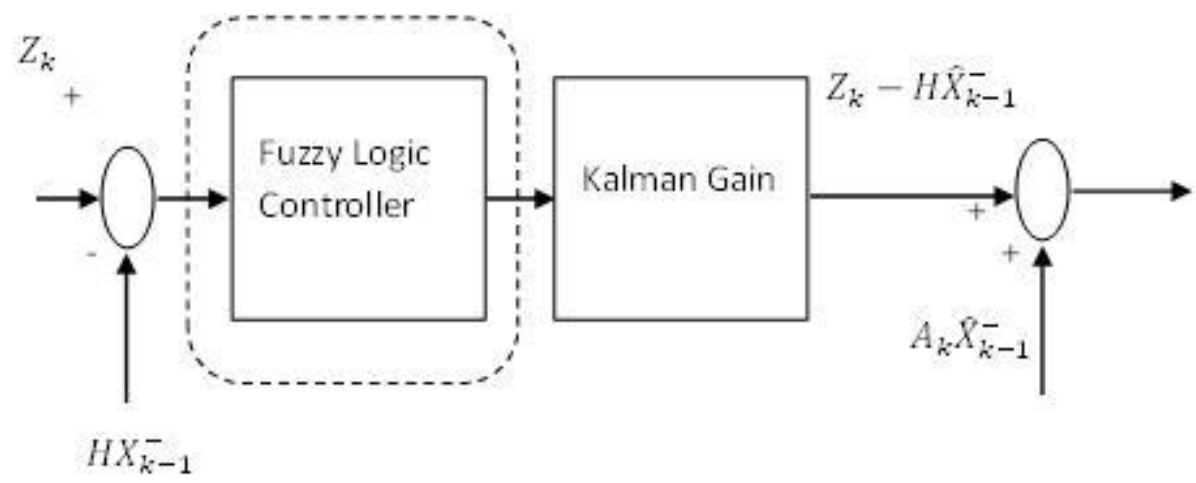

Figure 1. Modified measurement innovation calculation with the presence of fuzzy logic controller to the proposed system 


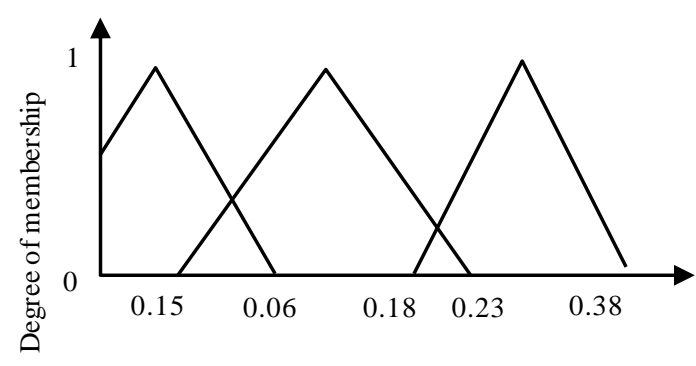

(a)

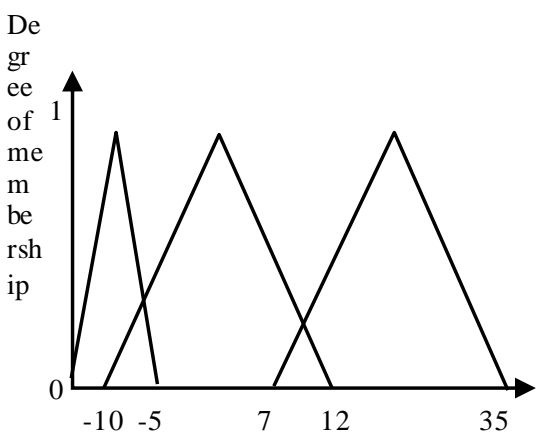

(c)

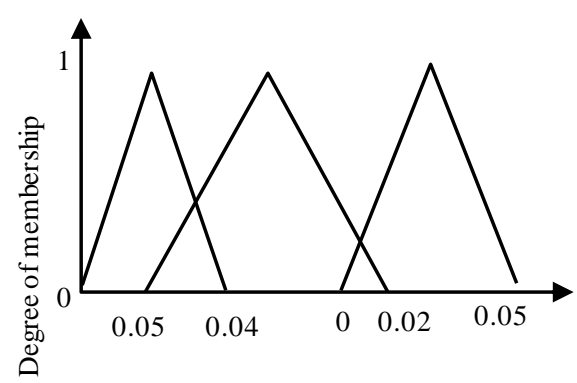

(b)

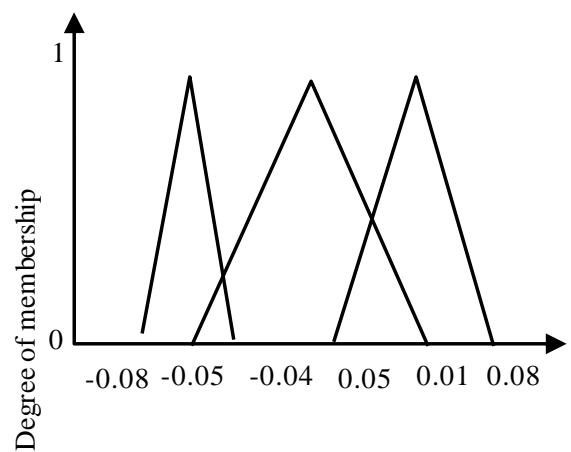

(d)

Figure 2. Input to the fuzzy logic (a) Measured relative angle (b) Measured relative distance. Output of the fuzzy logic (c) Calculated relative angle (d) Calculated relative distance

\section{Results and Analysis}

This section explains the results of research and at the same time is given the comprehensive discussion about our proposed technique. The simulation settings are described in Table 2 for reference. The parameters are chosen based on our previous experimental analysis. Global coordinate system is referred as the reference for the system to operate. The simulation considers two different environmental noises which are Gaussian and non-Gaussian noise to assess the EKF performance in both situations where suboptimal conditions take place during observation. The environment is also assume to be planar and mobile robot received all the observation during its measurements.

\subsection{EKF based SLAM with suboptimal conditions}

This subsection explains the effect of having an EKF as estimator in navigation especially in SLAM problem. It is undeniable, a normal EKF do the estimation nicely in Gaussian noise conditions but with a full computational cost of analysis. By doing some modifications on the state covariance update as shown in Table 1, the computational cost can be reduced hence produces faster computations. Figures 3 presents the performance of normal EKF and EKF with partial observability respectively. Mobile robot movements estimation as well as landmarks estimation determines that the EKF with partial observability conditions has better estimation. Remark that in partial observability, only certain landmarks are considered. Only three number of the landmarks are treated for reference while other landmarks are excluded 
from measurement innovation calculations. The mobile robot also holds very high confidence about its location compared to the normal conditions as depicted in Figure 3(b).

An interesting results happen when the environment conditions are changed to include non-Gaussian noise. If the mobile robot operates in non-Gaussian noise, then the results becomes not as promising as the previous case and this is shown in Figure 4. The estimation exhibits erroneous results especially for the landmarks estimation even when the mobile robot has higher confidence about its location(refer to Figure 4(b)). Notice that, the state covariance for suboptimal conditions are also producing lower uncertainties that eventually makes EKF becomes optimistics about its estimation [5]. For this case, minor modifications on the approach especially on measurement innovations must be made to guarantee better results.

Table 2. Simulation Parameters

\begin{tabular}{cc}
\hline Variable & Values \\
\hline Process Noise & $10 \mathrm{e}-7$ \\
Measurement Noise & Angular noise $=0.001$ \\
Distance noise $=0.001$ & Process noise \\
Random noise & (minimum -0.002, maximum 0.001$)$ \\
Measurement noise & (minimum -0.15, \\
maximum 0,01$)$ \\
Landmarks locations & $-20 ; 60 ;$ \\
& $-30 ; 120 ;$ \\
& $-200 ; 230 ;$ \\
& $-220 ; 160 ;$ \\
& $200 ; 160 ;$ \\
& $-190 ;-40 ;$ \\
& $170 ;-50 ;$ \\
& $20 ; 240 ;$ \\
& $-10 ;-200 ;$ \\
& $-80 ;-60 ;$ \\
Initial state covariance & $10 ; 130$ \\
Mobile Robot initial position & $3 ; 4] ;$ \\
\hline
\end{tabular}

\subsection{EKF based SLAM in Suboptimal Condition with Fuzzy Logic}

As shown in previous subsection, EKF becomes erroneous especially when nonGaussian noise exist during mobile robot observations. In order to overcome such inconsistency in estimation, Fuzzy Logic is proposed to correct the estimation. The Fuzzy Logic is then attached after the measurement innovation step to ensure that the updated state estimation becomes better and producing less error.

Preliminary results in Figure 5 have shown that by applying Fuzzy Logic, the landmarks estimation has become way better than the one being predicted by the EKF with suboptimal condition especially about its landmarks estimations. Additional simulations with different mobile robot movements are also being carried to obtain the consistency of the proposed technique. The results in figure 6 depicts that the proposed technique guarantees a good estimation can be achieved for different mobile robot movements compared to the normal EKF estimations. It is worth to mention that, if the mobile robot operates in different environment, Fuzzy Logic must be modified and re-design to suit with the new environment. Otherwise, the results can be not promising as expected. Based on the observations and literatures [9], the angle measurement plays an important role to guarantee good estimation. 


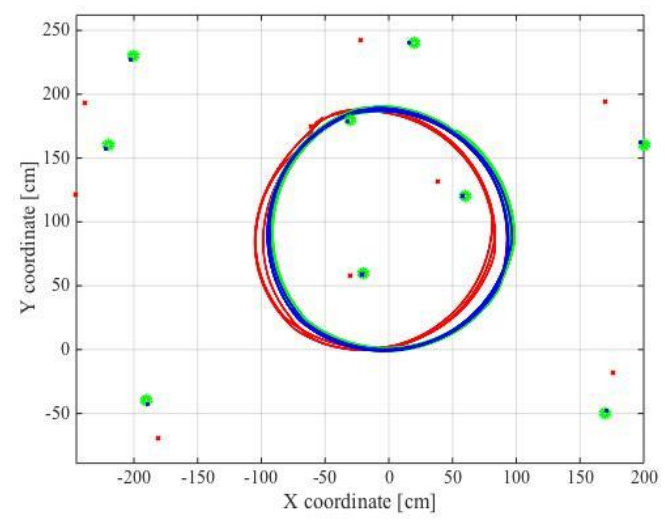

(a)

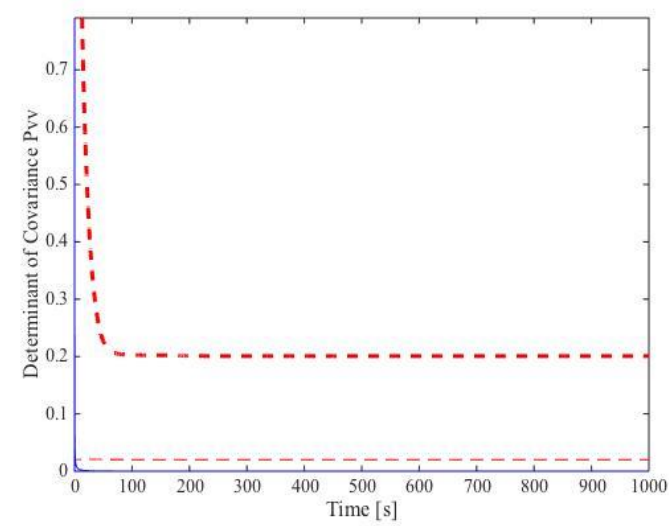

(b)

Figure 3 (a) Comparison between Normal EKF(red dash line) and EKF with suboptimal conditions(blue dash line).(b) State covariance performances for mobile robot and landmarks

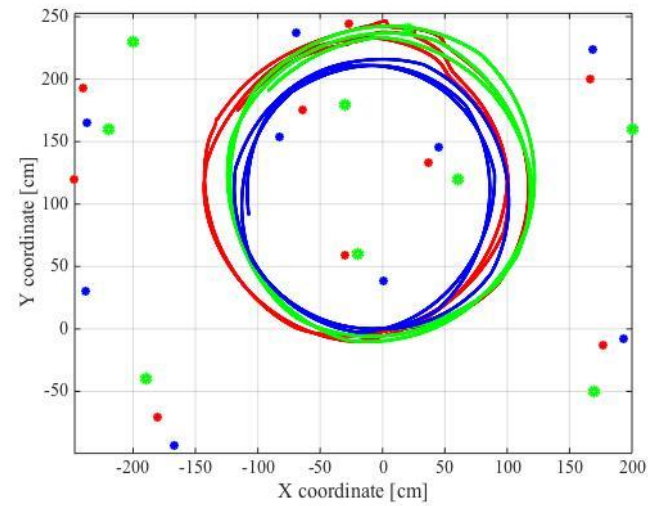

(a)

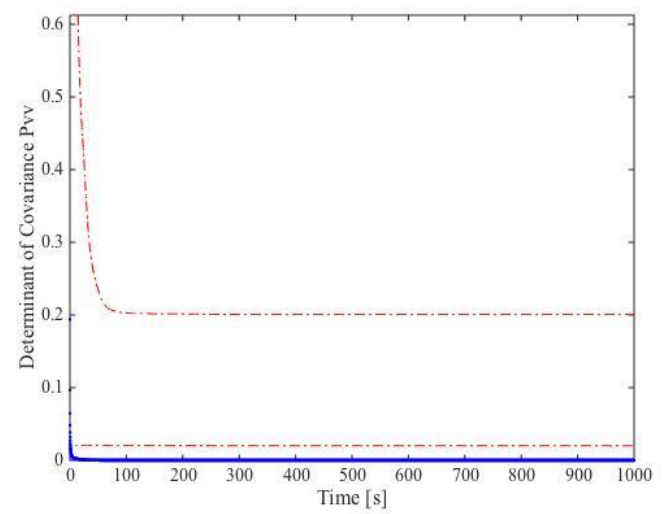

(b)

Figure 4 (a) Comparison between Normal EKF(red dash line) and EKF with suboptimal conditions(blue dash line) in non-Gaussian noise conditions. (b) State covariance performances for mobile robot and landmarks

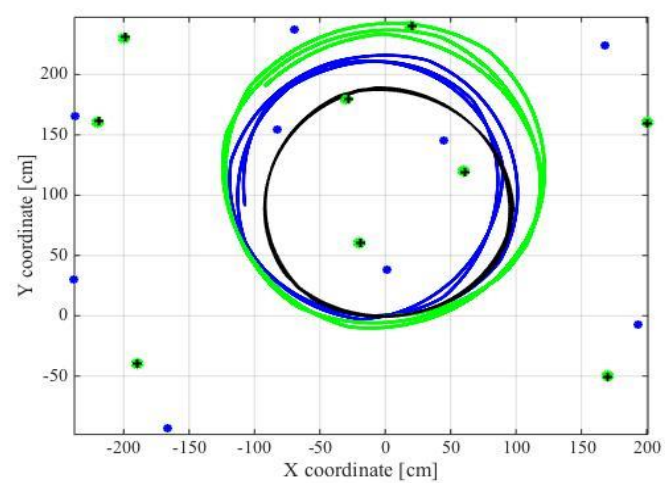

(a)

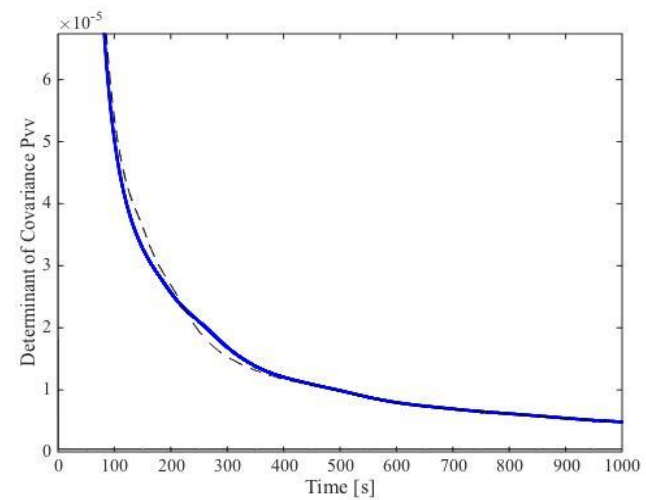

(b)

Figure 5. Comparison between suboptimal EKF(blue line) and Fuzzy EKF(black line) in nonGaussian noise conditions for both (a) mobile robot and landmarks estimation and (b) Updated state covariance performances 


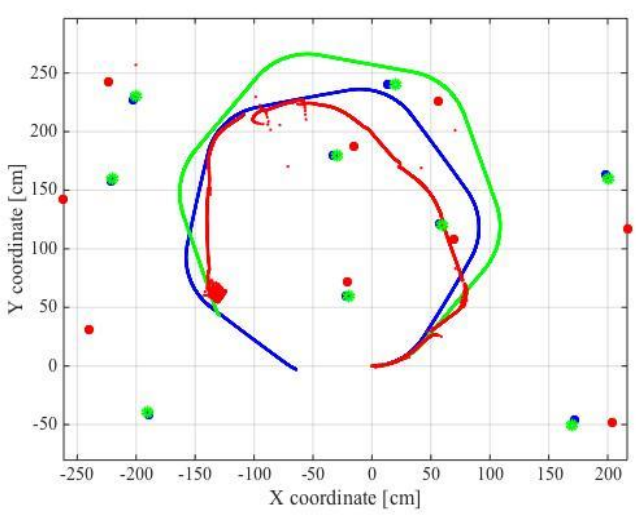

(a)

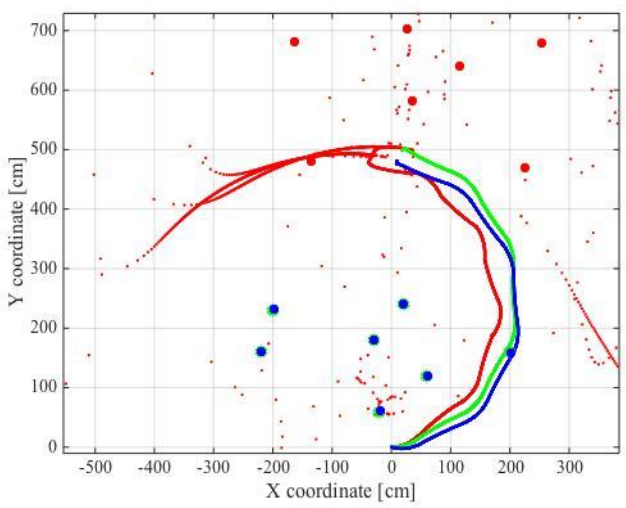

(b)

Figure 6. The performance of the proposed fuzzy EKF in suboptimal condition(blue) in various mobile robot (a), (b) movements compared to normal EKF performance

\section{Conclusion}

This paper has presented an analysis of Fuzzy Logic in Extended Kalman Filter navigation to prevent error in partial observability problem. Two different noise conditions are being analyzed to determine any inconsistency of the proposed method. Based on the results, the Fuzzy Logic technique can further improved the estimation results especially for the landmarks estimation for both gaussian and non-gaussian noise environments even with different mobile robot movements. Note that, as the proposed technique is only designed for a static landmarks conditions, the system performance can varies if more dynamic environment is taken into account for observation and require modifications on Fuzzy Logic design.

\section{Acknowledgements}

The author would like to thank Ministry of Higher Education and Universiti Malaysia Pahang for their continuous supports in this research through RDU160145 and RDU160379. Special thanks to Namerikawa Laboratory in Keio University for their participation and advises.

\section{References}

[1] S Thrun, et al. Probabilistic Robotic. 1st Edition. MIT Press. 2009; pp. 1-9.

TELKOMNIKA Vol. 16, No. 1, February 2018: $134-141$ 
[2] H Durrant Whyte, et al. Simultaneous Localization and Mapping: Part 1. IEEE Robotics \& Automation Magazine. 2006; 13(2): 99-110.

[3] J Andrade Cetto, et al. The Effects of Partial Observability in SLAM. IEEE International Conference on Robotics \& Automation, 2004; 1: 397-402.

[4] Guoquan P Huang, et al. On Filter Consistency of Discrete-Time nonlinear systems with partial-state measurements. in American Control Conference. 2013: 5468-5475.

[5] H.Ahmad, et al. EKF based Slam with FIM Inflation. 2011 8th Asian Control Conference(ASCC2011). 2011: 782-787.

[6] B.Noack, et al. Treatment of Biased and Dependent Sensor Data in Graph-Based SLAM. 18th International Conference on Information Fusion. 2015: 1862-1867.

[7] T Vidal-Valleja, et.al. Conditions for Suboptimal Filter Stability in SLAM. 2004 IEEE/RSJ International Conference on Intelligent Robots and Systems (IROS). 2004; 1:27-32.

[8] RC Smith, P Cheereman. On the representation and estimation of spatial uncertainty. Robol. Res, $1986 ; 5(4): 56-68$.

[9] MWMG Disranayake, P Newman, S Clark, HF Durrant-Whyte, M Csorba. A solution to the simultaneous localization and map building (SLAM) problem. IEEE Trsns. Robot. Automation. 2001; 17(3): 229-241.

[10] J Simanek, et al. Evaluation of the EKF-Based Estimation Architectures for Data Fusion in Mobile Robots. IEEE/ASME Transaction on Mechatronics. 2015;20(2): 985-990.

[11] D.Gualda. Partially Constrained Extended Kalman Filter for Navigation Inluding Mapping Information. IEEE Sensor Journal, 2016; 16(24): 9036-9046.

[12] T Zhang, et al. Convergence and Consistency Analysis for a 3-D Invariant-EKF SLAM. IEEE Robotic and Automatic Letters. 2017; 2(2): 733-740.

[13] SS Kia, et al. Cooperative Localization for Mobile Agents: A Recursive Decentralized Algorithm Based on Kalman-Filter Decoupling. IEEE Control System, 2016; 36(2): 86-101.

[14] M Negnevitsty. Artificial Intelligence: A Guide to Intelligent Systems. 3rd Edition, Addison-Wesley Pearson. 2011: 12-17. 